PROCEEDINGS OF THE

AMERICAN MATHEMATICAL SOCIETY

Volume 134, Number 7, Pages 1955-1961

S 0002-9939(05)08242-0

Article electronically published on December 16, 2005

\title{
HYPERCYCLIC SUBSPACES IN FRÉCHET SPACES
}

\author{
L. BERNAL-GONZÁLEZ
}

(Communicated by N. Tomczak-Jaegermann)

Dedicated to the memory of Professor Miguel de Guzmán, who died in April 2004

\begin{abstract}
In this note, we show that every infinite-dimensional separable Fréchet space admitting a continuous norm supports an operator for which there is an infinite-dimensional closed subspace consisting, except for zero, of hypercyclic vectors. The family of such operators is even dense in the space of bounded operators when endowed with the strong operator topology. This completes the earlier work of several authors.
\end{abstract}

\section{INTRODUCTION AND NOTATION}

Throughout this paper, the following standard notation will be used: $\mathbb{N}$ is the set of positive integers, $\mathbb{R}$ is the real line, and $\mathbb{C}$ is the complex plane. The symbols $\left(m_{k}\right),\left(n_{k}\right)$ will stand for strictly increasing sequences in $\mathbb{N}$. If $X, Y$ are (Hausdorff) topological vector spaces (TVSs) over the same field $\mathbb{K}=\mathbb{R}$ or $\mathbb{C}$, then $L(X, Y)$ will denote the space of continuous linear mappings from $X$ into $Y$, while $L(X)$ is the class of operators on $X$, that is, $L(X)=L(X, X)$. The strong operator topology (SOT) in $L(X)$ is the one where the convergence is defined as pointwise convergence at every $x \in X$.

A sequence $\left(T_{n}\right) \subset L(X, Y)$ is said to be universal or hypercyclic provided there exists some vector $x_{0} \in X$-called hypercyclic for the sequence $\left(T_{n}\right)$ - such that its orbit $\left\{T_{n} x_{0}: n \in \mathbb{N}\right\}$ under $\left(T_{n}\right)$ is dense in $Y$. It is obvious that if there is a hypercyclic sequence $\left(T_{n}\right) \subset L(X, Y)$, then $Y$ must be separable. In the (perhaps most interesting) case $X=Y$, an operator $T \in L(X)$ is called hypercyclic if the sequence $\left(T^{n}\right)$ of its iterates - that is, $T^{1}=T, T^{2}=T \circ T$ and so onis hypercyclic. See the excellent surveys [21] and 22] for concepts and results concerning universality.

In 1969 Rolewicz 33 proved that no finite-dimensional TVS $X$ supports a hypercyclic operator, and showed that any multiple $\lambda B(|\lambda|>1)$ of the backward shift $B:\left(\alpha_{0}, \alpha_{1}, \alpha_{2}, \ldots\right) \mapsto\left(\alpha_{1}, \alpha_{2}, \alpha_{3}, \ldots\right)$ on the sequence spaces $l_{p}(1 \leq p<\infty)$ or $c_{0}$ possesses a dense orbit, thereby exhibiting the first example of a hypercyclic operator on a Banach space. He asked in [33] whether any infinite-dimensional

Received by the editors October 6, 2004 and, in revised form, February 2, 2005.

2000 Mathematics Subject Classification. Primary 47A16; Secondary 47B37.

Key words and phrases. Hypercyclic operator, hypercyclic sequence, hypercyclic subspace, backward shift, Fréchet space.

The author was partially supported by the Plan Andaluz de Investigación de la Junta de Andalucía FQM-127 and by DGES Grant BFM2003-03893-C02-01.

(C)2005 American Mathematical Society Reverts to public domain 28 years from publication 
separable Banach space supports a hypercyclic operator. During the late nineties, this question was solved in the affirmative, independently, by Ansari [1] and the author [3] (the proof of Ansari is also applicable on a wide class of TVSs). Finally, in 1998 Bonet and Peris [13] gave the same affirmative answer for Fréchet spaces. We recall that an F-space is a complete and metrizable TVS, while a Fréchet space is a locally convex F-space. The proofs of [1, 3] and [13] are all based on a result of Salas [34, Theorem 3.3], who proved that when $\left(w_{n}\right)_{n \geq 0}$ is any bounded sequence of positive scalars, then the operator $I+B_{\omega}$-where $I$ denotes the identity operator and $B_{\omega}$ is the weighted backward shift generated by the weights $w_{n}$-is hypercyclic on $l_{p}(1 \leq p<\infty)$ or $c_{0}$. We point out that there exist separable complete locally convex spaces that do not support any hypercyclic operators; see [13, Proposition $6]$.

A natural problem that arises in this context is that of the size and the structure of the set $H C(T)$ of hypercyclic vectors for a hypercyclic operator $T$ on a TVS $X$. First, $H C(T)$ is dense because $T$ has dense range and $T^{n} x \in H C(T)$ for all $x \in H C(T)$ and all $n \in \mathbb{N}$. Second, if $X$ is Baire, metrizable and separable, then $H C(T)$ is even residual, that is, its complement in $X$ is of first category (more generally, if $X$ is Baire, $Y$ is metrizable separable and $\left(T_{n}\right) \subset L(X, Y)$ is a hypercyclic sequence, then its set $H C\left(\left(T_{n}\right)\right)$ of hypercyclic vectors is residual in $\left.X\right)$. It is evident that $H C(T)$ is not a linear space, but recently Wengenroth 36, has shown the existence of a dense linear manifold $M \subset X$ such that $M \backslash\{0\} \subset H C(T)$, thereby completing earlier work by Herrero [24, Bourdon [14] and Bès [8]; see also [4.

In a complementary line, the study of the existence of hypercyclic subspaces (the terminology is due to Chan [16]), that is, of infinite-dimensional closed subspaces $Z \subset X$ such that $Z \backslash\{0\} \subset H C(T)$ (or $\subset H C\left(\left(T_{n}\right)\right)$ if $\left(T_{n}\right)$ is a hypercyclic sequence) was initiated by Montes and the author [6] in 1995. They proved that if $G \subset \mathbb{C}$ is a domain that is not isomorphic to $\mathbb{C} \backslash\{0\}$, then there is a hypercyclic subspace for the sequence $\left(C_{\varphi_{n}}\right)$ of composition operators on $H(G)$ (= the Fréchet space of holomorphic functions on $G$, endowed with the compact-open topology) generated by a sequence $\left(\varphi_{n}\right)$ of automorphisms of $G$ acting properly discontinuously on $G$. This means that, given a compact set $K \subset G$, there is $n \in \mathbb{N}$ with $\varphi_{n}(K) \cap$ $K=\emptyset$. In particular, this applies on sequences of euclidean translations in $\mathbb{C}$ or of noneuclidean translations in the open unit disk $\mathbb{D}$. Later, Montes, León and González provided a series of papers ([29, 26], 27, [19]; see also [31, Section 2]) where some conditions for an operator on a Banach space $X$ to have a hypercyclic subspace are established. Among other results, they proved that if $T$ satisfies the Hypercyclicity Criterion (see Definition 2.1) for some $\left(n_{k}\right) \subset N$ and there exists an infinite-dimensional closed subspace $X_{0} \subset X$ such that $T^{n_{k}} x \rightarrow 0(k \rightarrow \infty)$ for all $x \in X_{0}$, then $T$ has a hypercyclic subspace. In [29, Theorem 3.4] it is shown that not every hypercyclic operator on a Hilbert space possesses a hypercyclic subspace. Nevertheless, every separable infinite-dimensional Banach space supports an operator that admits a hypercyclic subspace; see [26, Corollary 2.2].

Let us conclude the history of these recent results. Chan [15] gave an alternative simpler proof of the mentioned result of Montes and co-workers in the Hilbert space context. Later, Chan and Taylor [17 and, independently, Montes and Romero 30] extended the arguments of Chan to the setting of Banach spaces. In 2002, Chan [16] showed that hypercyclic operators on a Hilbert space are SOT-dense in the 
space of operators. Even more, he was able to prove that the family of operators on a (separable, infinite-dimensional) Hilbert space $X$ supporting a hypercyclic subspace is still SOT-dense in $L(X)$. Finally, Bès and Chan [9, Theorem 2] have recently noted that the hypercyclic operators on any (separable, infinite-dimensional) Fréchet space are SOT-dense.

In view of the aforementioned statements, the following problem arises naturally: Given an infinite-dimensional separable Fréchet space $X$, is the family of operators admitting a hypercyclic subspace SOT-dense in $L(X)$ ? The aim of this short note is to furnish a rather general answer by showing (see Section 2) that the denseness of such a family is guaranteed whenever $X$ admits a continuous norm. The space $H(G)$ is a typical example of a nonnormable space admitting a continuous norm.

\section{SUPPORTING OPERATORS WITH HYPERCYCLIC SUBSPACES}

Before giving the main result of the present paper (Theorem 2.5), we recall a notion which has turned out to be extremely useful in applications; see [18] and 21.

Definition 2.1. Assume that $X$ and $Y$ are separable F-spaces. Assume that $\left(T_{n}\right) \subset L(X, Y)$, that $T \in L(X)$ and that $\left(n_{k}\right) \subset \mathbb{N}$. We say that $\left(T_{n}\right)$ satisfies the Hypercyclicity Criterion (HC) (or the Universality Criterion) with respect to $\left(n_{k}\right)$ whenever there exist dense subsets $D_{1} \subset X, D_{2} \subset Y$ such that $T_{n_{k}} x \rightarrow 0(k \rightarrow \infty)$ for all $x \in D_{1}$, and for every $y \in D_{2}$ there is a sequence $\left(v_{k}\right) \subset X$ such that $v_{k} \rightarrow 0$ and $T_{n_{k}} v_{k} \rightarrow y(k \rightarrow \infty)$. The sequence $\left(T_{n}\right)$ satisfies the HC provided that the last property holds for some sequence $\left(n_{k}\right)$. Accordingly, $T$ is said to satisfy the $\mathrm{HC}$ (with respect to $\left(n_{k}\right)$ ) if the sequence $\left(T^{n}\right)$ of its iterates satisfies the $\mathrm{HC}$ (with respect to $\left(n_{k}\right)$, respectively).

If $\left(T_{n}\right)$ satisfies the HC, then it is hypercyclic; see [21. The reader is referred to [7, 11], [5] and [2] for equivalent properties to the HC.

We will make use of four auxiliary results. The first one is due to Grivaux 20, Theorem 1.3 and Remark 1.4]. It is in fact a modification of [34, Theorem 3.3] and reads as follows.

Lemma 2.1. Let $X$ be one of the sequence spaces $l_{p}(1 \leq p<\infty)$ or $c_{0}$, and let $\left(w_{n}\right)_{n \geq 0}$ be any bounded sequence of positive scalars. Consider the operator $S$ defined on $X$ by

$$
S\left(\alpha_{0}, \alpha_{1}, \alpha_{2}, \alpha_{3}, \alpha_{4}, \alpha_{5}, \ldots\right)=\left(w_{0} \alpha_{1}, w_{1} \alpha_{3}, w_{2} \alpha_{5}, \ldots\right) .
$$

Then the operator $I+S$ is hypercyclic on $X$. In fact, it satisfies the HC.

The second auxiliary statement is a very useful one due to Martínez-Giménez and Peris [28, Lemma 2.1]. It is a stronger version of the so-called Hypercyclic Comparison Principle due to J.H. Shapiro [35, p. 111].

Lemma 2.2. Assume that $X, Y$ are F-spaces, that $T \in L(X), \widetilde{T} \in L(Y), Q \in$ $L(Y, X)$, that $Q$ has dense range and that $Q \widetilde{T}=T Q$. If $\widetilde{T}$ satisfies the $H C$, then $T$ does also.

The third auxiliary statement is a modified version of Theorem 3.5 of the very recent paper [12] by Bonet, Martínez-Giménez and Peris. In our lemma, condition (iii) below has replaced the more restrictive one " $\lim _{k \rightarrow \infty} T_{m_{k}} x=0$ for each $x \in$ $X_{0}$ " given in [12, Theorem 3.5]. 
Lemma 2.3. Assume that $\left(m_{k}\right) \subset \mathbb{N}$, that $X$ and $Y$ are Fréchet spaces and that $\left(S_{n}\right) \subset L(X, Y)$. Suppose that the following properties are satisfied:

(i) The space $X$ admits a norm which is continuous.

(ii) The sequence $\left(S_{n}\right)$ satisfies the $H C$ with respect to $\left(m_{k}\right)$.

(iii) There exists a closed infinite-dimensional subspace $X_{0} \subset X$ such that, for every $x \in X_{0}$, the sequence $\left(S_{m_{k}} x\right)$ converges in $Y$.

Then $\left(S_{m_{k}}\right)$ (hence $\left(S_{n}\right)$ ) admits a hypercyclic subspace.

Proof. Exactly as in [12, proof of Theorem 3.5], conditions (i) and (ii) allow us to assert the existence of an operator $K$ on $X$ such that $\left\{S_{m_{k}} K x: k \in \mathbb{N}\right\}$ is dense in $Y$ for all $x \in X \backslash\{0\}$ and, in addition, the operator $I+K$ has closed range and finite-dimensional kernel. Therefore $X_{1}:=(I+K)\left(X_{0}\right)$ is a closed infinitedimensional subspace of $X$. If $x_{1} \in X_{1} \backslash\{0\}$, then there exists $x_{0} \in X_{0} \backslash\{0\}$ such that $x_{1}=x_{0}+K x_{0}$. Let $u\left(x_{0}\right):=\lim _{k \rightarrow \infty} S_{m_{k}} x_{0} \in Y$. Fix $y \in Y$ and let $\left(n_{k}\right)$ be a subsequence of $\left(m_{k}\right)$ satisfying $S_{n_{k}} K x_{0} \rightarrow y-u\left(x_{0}\right)$ as $k \rightarrow \infty$. Hence, by linearity,

$$
S_{n_{k}} x_{1}=S_{n_{k}} x_{0}+S_{n_{k}} K x_{0} \rightarrow u\left(x_{0}\right)+\left(y-u\left(x_{0}\right)\right)=y \quad(k \rightarrow \infty) .
$$

In other words, the sequence $\left\{S_{m_{k}} x_{1}: k \in \mathbb{N}\right\}$ is dense in $Y$. Consequently, $X_{1}$ is a hypercyclic subspace for $\left(S_{m_{k}}\right)$.

The fourth and last auxiliary result is a nice one due to Hadwin, Nordgren, Radjavi and Rosenthal ([23, see also [10, Theorem 1 and the note following it]), and it was cleverly used by Bès and Chan in [10, Corollary 6] to give a new proof of [9, Theorem 2] together with some new results.

Lemma 2.4. Let $X$ be an infinite-dimensional locally convex space, and let $T$ in $L(X)$ be fixed. The following statements are equivalent:

(i) The set of conjugates $\left\{S T S^{-1}: S\right.$ invertible $\}$ of $T$ is SOT-dense in $L(X)$.

(ii) For all $n \in \mathbb{N}$, there exist $h_{1}, \ldots, h_{n}$ in $X$ so that the set $\left\{h_{1}, \ldots, h_{n}\right.$, $\left.T h_{1}, \ldots, T h_{n}\right\}$ is linearly independent.

If $X$ is a TVS, then the linear span of a subset $A$ of $X$ is denoted by $\operatorname{span}(A)$. If $A$ is an absolutely convex subset of $X$ and $p_{A}$ is the Minkowski functional of $A$, $X_{A}$ will stand for $\operatorname{span}(A) / \operatorname{ker} p_{A}$ endowed with the norm induced by $p_{A}$. The topological dual of $X$ will be denoted by $X^{\prime}$. We are now ready to establish our assertion, which concludes the paper.

Theorem 2.5. Suppose that $X$ is a separable infinite-dimensional Fréchet space admitting a continuous norm. Then $X$ supports an operator which possesses a hypercyclic subspace. Even more, the family of such operators is SOT-dense in $L(X)$.

Proof. The second assertion of the theorem is obtained from the first one together with Lemma 2.4: Just take into account that the family under consideration is invariant under conjugation, and that every hypercyclic operator has at least one dense (so linearly independent, because $X$ is infinite dimensional) orbit $\left\{x_{0}, T x_{0}, T^{2} x_{0}, \ldots\right\}$; then Lemma 2.4 applies if we choose $h_{j}:=T^{2 j-2} x_{0}$ $(j=1, \ldots, n ; n \in \mathbb{N})$.

Hence it is enough to exhibit an operator $T \in L(X)$ possessing a hypercyclic subspace. Our approach takes advantage of the clever method given by Bonet and Peris in [13, Lemmas 2-3]. 
It is known that the countable product of lines $\omega:=\mathbb{K}^{\mathbb{N}}$, endowed with the product topology, is a Fréchet space, but it does not admit a continuous norm, so $X \neq \omega$. Then by [13, Lemma 2 and proof of Theorem 1] there are sequences $\left(x_{n}\right)_{n \geq 0} \subset X$ and $\left(f_{n}\right)_{n \geq 0} \subset X^{\prime}$ satisfying the following conditions:

(a) $\left(x_{n}\right)$ converges to 0 in $X$, and the closed absolutely convex hull $B$ of $\left(x_{n}\right)$ satisfies that $X_{B}$ is a Banach space which is dense in $X$.

(b) $\left(f_{n}\right)$ is $X$-equicontinuous in $X^{\prime}$.

(c) $f_{m}\left(x_{n}\right)=0$ if $m \neq n$ and $\left\{f_{n}\left(x_{n}\right): n \geq 0\right\} \subset(0,1)$.

Consider the operator $T$ on $X$ defined as $T:=I+S$, where

$$
S x:=\sum_{n=0}^{\infty} \frac{f_{2 n+1}(x)}{2^{n}} x_{n} \quad(x \in X) .
$$

Exactly as in [13, proof of Lemma 3], we obtain that

$$
B=\left\{\sum_{n=0}^{\infty} \alpha_{n} x_{n}: \sum_{n=0}^{\infty}\left|\alpha_{n}\right| \leq 1\right\}
$$

and that the mapping

$$
Q: \alpha=\left(\alpha_{n}\right)_{n \geq 0} \in l_{1} \mapsto \sum_{n=0}^{\infty} \alpha_{n} x_{n} \in X_{B}
$$

is linear, continuous and surjective. By Lemma 2.1, the operator

$$
\widetilde{T}:\left(\alpha_{n}\right)_{n \geq 0} \in l_{1} \mapsto\left(\alpha_{0}+f_{0}\left(x_{0}\right) \alpha_{1}, \alpha_{1}+\frac{f_{1}\left(x_{1}\right)}{2} \alpha_{3}, \alpha_{2}+\frac{f_{2}\left(x_{2}\right)}{2^{2}} \alpha_{5}, \ldots\right) \in l_{1}
$$

satisfies the $\mathrm{HC}$, since the weights $w_{n}:=\frac{f_{n}\left(x_{n}\right)}{2^{n}}$ are positive and form a bounded sequence.

Let us show that $T$ also satisfies the HC. If we consider $Q$ as a mapping $Q$ : $l_{1} \rightarrow X$, then $Q$ is also continuous. Indeed, such a mapping is the composition of $Q: l_{1} \rightarrow X_{B}$ with the canonical inclusion $X_{B} \rightarrow X$. This inclusion is (linear and) continuous, because if $\left\{u_{k}:=\sum_{n=0}^{\infty} \alpha_{n k} x_{n}\right\}_{k \in \mathbb{N}} \subset X_{B}$ is a sequence tending to zero in the topology of $X_{B}$, then it also tends to zero in the topology of $E$. Let us explain why this is so. By (1) we have that $\lambda B=\left\{\sum_{n=0}^{\infty} \alpha_{n} x_{n}: \sum_{n=0}^{\infty}\left|\alpha_{n}\right| \leq \lambda\right\}$ for all $\lambda>0$. Due to (c), the series expansion $x=\sum_{n=0}^{\infty} \alpha_{n} x_{n}$ of each $x \in X_{B}$ in terms of some sequence $\left(\alpha_{n}\right) \subset \mathbb{K}$ is unique. So if $\lambda>0$ and $x \in \lambda B$ we get $\sum_{n=0}^{\infty}\left|\alpha_{n}\right| \leq \lambda$. Therefore $\sum_{n=0}^{\infty}\left|\alpha_{n}\right| \leq p_{B}(x)$. But $\lim _{k \rightarrow \infty} p_{B}\left(u_{k}\right)=0$, whence $\lim _{k \rightarrow \infty} \sum_{n=0}^{\infty}\left|\alpha_{n k}\right|=0$. On the other hand, $X$ is locally convex, so in order to show that $\left(u_{k}\right)$ tends to zero in $X$ it is enough to prove that $u_{k} \rightarrow 0$ in the Mackey topology of $X$, that is, that $\lim _{k \rightarrow \infty} \sup _{\varphi \in A}\left|\varphi\left(u_{k}\right)\right|=0$ for every equicontinuous subset $A \subset X^{\prime}$ (see for instance [25, Proposition 7, pages 200-201]). Since $\left(x_{n}\right)$ is bounded (because $x_{n} \rightarrow 0$ ) in $X$, it holds that if $A$ is equicontinuous, then there is a constant $K \in(0,+\infty)$ such that $\sup _{\varphi \in A, n \geq 0}\left|\varphi\left(x_{n}\right)\right| \leq K$. Thus,

$$
\begin{array}{r}
\sup _{\varphi \in A}\left|\varphi\left(u_{k}\right)\right|=\sup _{\varphi \in A}\left|\varphi\left(\sum_{n=0}^{\infty} \alpha_{n k} x_{n}\right)\right| \\
\leq \sum_{n=0}^{\infty}\left|\alpha_{n k}\right| \sup _{\substack{\varphi \in A \\
n \geq 0}}\left|\varphi\left(x_{n}\right)\right| \leq K \sum_{n=0}^{\infty}\left|\alpha_{n k}\right| \rightarrow 0 \quad(k \rightarrow \infty),
\end{array}
$$


from which one derives that the left-hand side tends to zero as $k \rightarrow \infty$, as desired. Consequently, $Q: l_{1} \rightarrow X$ is linear and continuous. In addition, it has dense range (due to $Q\left(l_{1}\right)=X_{B}$ and (a)) and $T Q=Q \widetilde{T}$ on $l_{1}$. Recall that $T \in L(X), \widetilde{T} \in L\left(l_{1}\right)$ and that $\widetilde{T}$ satisfies the HC. This and Lemma 2.2 (with $Y:=l_{1}$ ) drive us to assert that $T$ satisfies the $\mathrm{HC}$.

On the other hand, the restriction of the operator $\widetilde{T}$ to the subspace $Z:=\{\alpha \in$ $l_{1}: \alpha_{2 n+1}=0$ for all $\left.n \geq 0\right\}$ is the identity operator. Consider the linear manifold $Z_{0}:=Q(Z) \subset X_{B} \subset X$. Since $T Q=Q \widetilde{T}$ on $l_{1}$, the fact that the restriction of $\widetilde{T}$ to $Z$ is the identity yields that $T$ is the identity on $Z_{0}$, i.e. $T x=x$ for all $x \in Z_{0}$. By continuity, we get $T x=x$ for all $x \in X_{0}$, where

$$
X_{0}:=\operatorname{closure}_{X}\left(Z_{0}\right) \text {. }
$$

From (c) one easily derives that the sequence $\left(x_{n}\right)$ is linearly independent. Therefore $\operatorname{span}\left(\left\{x_{2 n}: n \geq 0\right\}\right)$ is infinite dimensional, hence by (2) and by the inclusions $\operatorname{span}\left(\left\{x_{2 n}: n \geq 0\right\}\right) \subset Z_{0} \subset X_{0}$ we obtain that $X_{0}$ is a closed infinite-dimensional subspace of $X$.

Finally, if $x \in X_{0}$, then $T x=x$, so $S_{n} x=x$ for all $n \in \mathbb{N}$ and all $x \in X_{0}$, where we have set $S_{n}:=T^{n}(n \in \mathbb{N})$. Hence, trivially, $\lim _{n \rightarrow \infty} S_{n} x$ exists $(=x)$ for every $x \in X_{0}$. Therefore $\left(S_{m_{k}} x\right)$ converges in $X$ for each $x \in X_{0}$, where $\left(m_{k}\right) \subset \mathbb{N}$ is a sequence with respect to which $T$ - or, which is the same, the sequence $\left(S_{n}\right)$ satisfies the HC. An application of Lemma 2.3 with $Y:=X$ concludes the proof.

\section{ACKNOWLEDGEMENT}

I am grateful to the referee for useful comments and for letting me know that the first part of the statement of our Theorem 2.5 has also been obtained, independently, by Henrik Petersson in his recent manuscript 32 .

\section{REFERENCES}

[1] S.I. Ansari, Existence of hypercyclic operators on topological vectors spaces, J. Funct. Anal. 148 (1997), 384-390. MR.1469346 (98h:47028a)

[2] T. Bermúdez, A. Bonilla and A. Peris, On hypercyclicity and supercyclicity criteria Bull. Austral. Math. Soc. 70 (2004), 45-54. MR2079359 (2005d:47014)

[3] L. Bernal-González, On hypercyclic operators on Banach spaces, Proc. Amer. Math. Soc. 127 (1999), 1003-1010. MR1476119 (99f:47010)

[4] L. Bernal-GonzÁlez, Universal images of universal elements, Studia Math. 138 (2000), 241-250. MR1758857 (2001f:47002)

[5] L. Bernal-GonzÁlez and K.-G. Grosse-Erdmann, The hypercyclicity criterion for sequences of operators, Studia Math. 157 (2003), 17-32. MR1980114 (2003m:47013)

[6] L. Bernal-GonzÁlez and A. Montes-Rodríguez, Non-finite dimensional closed of universal functions for composition operators, J. Approx. Theory 82 (1995), 375-391. MR 1348728 (96f:30034)

[7] J. BÈs, Three problems on hypercyclic operators, Thesis, Kent State University, 1998.

[8] J. Bès, Invariant manifolds of hypercyclic vectors for the real scalar case, Proc. Amer. Math. Soc. 127 (1999), 1801-1804. MR1485460 (99i:47002)

[9] J.P. BÈs and K. ChAn, Denseness of hypercyclic operators on a Fréchet space, Houston J. Math. 29 (2003), 195-206. MR1952504 (2003j:47006)

[10] J.P. BÈs and K. ChAN, Approximation by chaotic operators and by conjugate classes, J. Math. Anal. Appl. 284 (2003), 206-212. MR1996128(2004j:47014)

[11] J.P. BÈs and A. Peris, Hereditarily hypercyclic operators, J. Funct. Anal. 167 (1999), 94113. MR1710637(2000f:47012)

[12] J. Bonet, F. Martínez-Giménez and A. Peris, Universal and chaotic multipliers on spaces of operators, J. Math. Anal. Appl. 297 (2004), 599-611. MR2088683 
[13] J. Bonet and A. Peris, Hypercyclic operators on non-normable Fréchet spaces, J. Funct. Anal. 159 (1998), 587-596. MR1658096 (99k:47044)

[14] P.S. Bourdon, Invariant manifolds of hypercyclic operators, Proc. Amer. Math. Soc. 118 (1993), 845-847. MR.1148021 (93i:47002)

[15] K.C. Chan, Hypercyclicity of the operator algebra for a separable Hilbert space J. Operator Theory 42 (1999), 231-244. MR 1716973 (2000i:47066)

[16] K.C. Chan, The density of hypercyclic operators on a Hilbert space, J. Operator Theory 47 (2002), 131-143. MR.1905817 (2003c:47011)

[17] K.C. Chan and R.D. TAYlor, Hypercyclic subspaces of a Banach space, Integr. Equ. Oper. Theory 41 (2001), 381-388. MR1857797|(2002g:47011)

[18] G. Godefroy and J. H. Shapiro, Operators with dense, invariant, cyclic vector manifolds, J. Funct. Anal. 98 (1991), 229-269. MR.1111569 (92d:47029)

[19] M. González, F. León-Sanvedra and A. Montes-Rodríguez, Semi-Fredholm theory: Hypercyclic and supercyclic subspaces, Proc. London Math. Soc. 81 (2000), 169-189. MR:1757050 (2001g:47013)

[20] S. Grivaux, Hypercyclic operators with an infinite dimensional closed subspace of periodic points, Rev. Mat. Complutense 16 (2003), 383-390. MR2032923 (2004m:47018)

[21] K.G. Grosse-Erdmann, Universal families and hypercyclic operators, Bull. Amer. Math. Soc. (N.S.) 36 (1999), 345-381. MR1685272(2000c:47001)

[22] K.G. Grosse-Erdmann, Recent developments in hypercyclicity, Rev. R. Acad. Cien. Serie A. Mat. 97 (2003), 273-286. MR2068180 (2005c:47010)

[23] D.W. Hadwin, E.A. Nordgren, H. Radjavi and P. Rosenthal, Most similarity orbits are strongly dense, Proc. Amer. Math. Soc. 76 (1979), 250-252. MR0537082 (80i:47029)

[24] D.A. Herrero, Limits of hypercyclic and supercyclic operators, J. Funct. Anal. 99 (1991), 179-190. MR 1120920 (92g:47026)

[25] J. Horváth, Topological vector spaces and distributions, Vol. 1, Addison-Wesley, Reading, 1966. MR0205028 (34:4863)

[26] F. León-SaAvedra and A. Montes-Rodríguez, Linear structure of hypercyclic vectors, J. Funct. Anal. 148 (1997), 524-545. MR1469352 (98h:47028b)

[27] F. LeÓN-SAavedra and A. Montes-Rodríguez, Spectral theory and hypercyclic subspaces, Trans. Amer. Math. Soc. 353 (2000), 247-267. MR.1783790 (2001h:47008)

[28] F. Martínez-Giménez and A. Peris, Chaos for backward shift operators, Int. J. Bifurcation and Chaos 12 (2002), 1703-1715. MR1927407 (2003h:47056)

[29] A. Montes-Rodríguez, Banach spaces of hypercyclic vectors, Michigan Math. J. 43 (1996), 419-436. MR1420585 (98g:47027)

[30] A. Montes-Rodríguez and M.C. Romero-Moreno, Supercyclicity in the operator algebra, Studia Math. 150 (2002), 201-213. MR.1891844 (2003b:47016)

[31] A. Montes-Rodríguez and H.N. Salas, Supercyclic subspaces, Bull. London Math. Soc. 35 (2003), 721-737. MR2000019 (2004d:47022)

[32] H. Petersson, Hypercyclic subspaces for Fréchet space operators, preprint 2004.

[33] S. Rolewicz, On orbits of elements, Studia Math. 32 (1969), 17-22. MR0241956 (39:3292)

[34] H. Salas, Hypercyclic weighted shifts, Trans. Amer. Math. Soc. 347 (1995), 993-1004. MR.1249890(95e:47042)

[35] J.H. ShAPIRO, Composition operators and classical function theory, Springer-Verlag, New York, 1993. MR1237406 (94k:47049)

[36] J. Wengenroth, Hypercyclic operators on non-locally convex spaces, Proc. Amer. Math. Soc. 131 (2003), 1759-1761. MR1955262 (2003j:47007)

Departamento De Análisis Matemático, Facultad De Matemáticas, Apdo. 1160, Avenida Reina Mercedes, 41080 Sevilla, Spain

E-mail address: lbernal@us.es 